\title{
Cisplatin binds to pre-miR-200b and impairs its processing to mature microRNA
}

\author{
R. MEZENCEV*, R. M. WARTELL \\ Georgia Institute of Technology, School of Biological Sciences, 310 Ferst Dr, Atlanta, GA 30332, USA \\ ${ }^{*}$ Correspondence: roman.mezencev@biosci.gatech.edu
}

Received January 16, 2017 / Accepted June 19, 2017

\begin{abstract}
Cisplatin is an important anticancer drug with a complex mode of action, a variety of possible targets, and numerous resistance mechanisms. While genomic DNA has traditionally been considered to be its most critical anticancer target, several lines of evidence suggest that various RNAs and other biomolecules may play a role in its anticancer mode of action. In this report we demonstrate that cisplatin modifies pre-miR-200b, impairs its processing to mature miRNA, and decreases miR-200b expression in ovarian cancer cells. Considering the role of miR-200b in epithelial-to-mesenchymal transition and cancer chemosensitivity, cisplatin-induced modification of pre-miR-200b and subsequent deregulation of mature miR-200b may, depending on cell context, limit anticancer activity of this important anticancer drug. More generally, precursor miRNAs may be important targets of cisplatin and play a role in this drug's anticancer activity or modulate cell responses to this drug.
\end{abstract}

Key words: cisplatin, pre-miRNA, microRNA, transplatin, miR-200

Cisplatin is a traditional anticancer drug that has been widely used for treatment of various solid tumors, and served as a lead compound for development of second (e.g. carboplatin), third (e.g. oxaliplatin) and fourth generation (satraplatin) platinum-based anticancer drugs. Given that these newer analogs have not demonstrated higher efficacy and better toxicity profiles in all relevant malignancies, cisplatin remains to be an important drug in clinical oncology. In addition, this compound remains interesting for experimental cancer and pharmacology research, due to its complex mode of action, variety of possible targets and numerous resistance mechanisms, which are often shared with newer platinumbased anticancer agents [1]. A more complete understanding of these molecular mechanisms can help in the development of more successful cisplatin analogs or refine clinical use of cisplatin so that its remarkable success in the treatment of testicular germ cell tumors is reproduced in other cancers.

Genomic DNA has been traditionally recognized as cisplatin's most critical anticancer target, in which it produces a variety of lesions, including monoadducts, intrastrand and interstrand cross-links and DNA-protein cross-links. Nevertheless, evidence suggests that numerous non-DNA targets, including various types of RNA, can also be involved in its mode of action (reviewed in [2]). Among them, microRNAs (miRNAs) may represent the most significant RNA targets of cisplatin, considering their ability to regulate numerous genes, including genes involved in cancer-related signaling pathways [3]. Consequently, identification of miRNA targets of cisplatin may be essential for better understanding the mode of action of this anticancer drug.

According to the most recent release of miRNA Sequence Database and Registry (miRBase 21) [4], there have been 2588 mature human miRNA species identified so far. Among them, members of the miR-200 family have been shown to play critical roles in cancer progression and drug resistance through regulation of epithelial-to-mesenchymal transition (EMT), cancer stem cell renewal, apoptosis and expression of drug resistance genes (reviewed in [5]). MIR-200b, in particular, has been shown to repress EMT [6] and metastatic competence of cancer cells [7], and differentially influence chemosensitivity in a cell context-dependent and drugdependent way $[8,9]$. In this report, we demonstrate that cisplatin interacts with pre-miR-200b (the hairpin precursor of miR-200 family member miR-200b, Supplemental Material, Figure S1), impairs its processing to mature miR-200b, and decreases its expression in ovarian cancer cells. We also show 
that these effects are not produced by transplatin, an inactive geometric isomer of cisplatin. Our results indicate for the first time that cisplatin directly interacts with a human precursor miRNA and suggest that this type of interaction may play an important role in the anticancer activity of cisplatin.

\section{Materials and methods}

Reagents. Cisplatin (Supplemental Material, Figure S1A) and transplatin (Supplemental Material, Figure S1B) were procured from Sigma-Aldrich (catalog No. 479306-1G and P1525-1G), dissolved in $0.9 \% \mathrm{NaCl}$ at final concentration $0.5 \mathrm{mg} / \mathrm{mL}$ and stored in the dark at $4{ }^{\circ} \mathrm{C}$.

Preparation of pre-miR-200b. A DNA template for in vitro transcription of pre-miR-200b (Supplemental Material, Figure S1C) was prepared from overlapping oligonucleotides (Supplemental Material, Table S1) using previously described recursive PCR procedure [10]. The DNA was purified by gel electrophoresis and PCR amplified. The purified PCR product was digested with HindIII and EcoRI restriction enzymes and cloned into pUC19 plasmid, which was subsequently used to transform E. coli strain XL-1 Blue. Clones containing plasmid with the DNA insert (pUC19-200b) were selected and blue-white screened on LB-ampicillin plates with X-gal (5-bromo-4-chloro-3-indolyl-beta-Dgalacto-pyranoside). The E. coli cells were propagated in LB broth with ampicillin $(100 \mu \mathrm{g} / \mathrm{mL})$ and plasmid was isolated using PureYield Midiprep System (Promega). The sequence of the DNA insert was verified by Sanger sequencing. HindIII linearized plasmid was used as template for in vitro transcription using MEGAscript ${ }^{\circledast}$ T7 Transcription Kit (Ambion). Transcribed pre-miR-200b was purified by gel chromatography on NAP5 column (G25 Sephadex) and concentrated with the Amicon Ultra NMWL $3 \mathrm{kDa}$ centrifugal filter (EMD Millipore). Transcribed pre-miR$200 \mathrm{~b}$ was characterized by $10 \%$ PAGE/8M urea (Supplemental Material, Figure S2). Concentration of RNA was determined using Quant-it ssDNA Assay Kit (Invitrogen).

Platination reactions. Reactions of pre-miR-200b with cisplatin or transplatin were performed in $0.05 \mathrm{M}$ potassium phosphate buffer (pH 7.4) with $0.05 \mathrm{mM}$ EDTA and $32 \mathrm{mM}$ $\mathrm{Cl}^{-}$at final pre-miRNA and drug concentrations of $0.42 \mu \mathrm{M}$ $(12.5 \mathrm{ng} / \mathrm{mL}$ ) and $3.85 \mu \mathrm{M}$, respectively (Nucleotide: $\mathrm{Pt}=10: 1$ ). Reactions proceeded for 48 hours at $37^{\circ} \mathrm{C}$. An un-reacted pre-miR-200b sample was incubated in parallel with the platination reactions and used as the control.

RNA melting analysis. UV absorbance spectroscopy is commonly used to examine the temperature dependent unfolding behavior of nucleic acids and detect ligandinduced structural perturbations. This method, which has been previously employed to study various types of nucleic acids, including RNA hairpins [11], is based on the hyperchromicity resulting from the unstacking of the nucleotide bases upon disruption of base-pairing. Reaction mixtures were used directly for melting analysis. Melting curves were recorded using a CARY $1 \mathrm{E}$ UV-Visible spectrophotometer with a Peltier temperature controller. Sample absorbance was measured in a $1 \mathrm{~cm}$ path length cells at $260 \mathrm{~nm}$ every $0.2^{\circ} \mathrm{C}$ as the temperature changed from $25^{\circ} \mathrm{C}$ to $95^{\circ} \mathrm{C}$ at a rate of $0.5^{\circ} \mathrm{C} / \mathrm{min}$. The temperature dependent absorbance data was normalized to the absorbance value at $25^{\circ} \mathrm{C}$ (i.e., $\mathrm{A}(\mathrm{T}) / \mathrm{A}_{25}$ ) and then smoothed over a $4^{\circ} \mathrm{C}$ window using the Loess method with a first order polynomial. The first derivative melting curve $\left([\mathrm{dA}(\mathrm{T}) / \mathrm{dT}] / \mathrm{A}_{25}\right)$ was computed by a 13 point running average of adjacent numerical derivatives (SigmaPlot Inc). Three independent melting experiments gave reproducible transitions.

Dicer digestion. Pre-miR-200b and its platinated products were processed using Dicer siRNA Generation Kit-T510001 (Genlantis). Each $20 \mu \mathrm{L}$ reaction contained $250 \mathrm{ng}$ pre-miRNA, $1 \mathrm{mM}$ ATP, $2.5 \mathrm{mM} \mathrm{MgCl}_{2}$ and 1 Unit of recombinant Dicer (or no Dicer control) and the reactions were allowed to proceed for 17 hours. Prior to Dicer digestion, miRNA-200b or its platinated products were washed with water and concentrated using Amicon Ultra Centrifugal Filter NMWL 10K (EMD Millipore). Digestion products were resolved by $12 \%$ native PAGE or $12 \%$ PAGE/8M urea and visualized using SYBR $^{\oplus}$ Gold Nucleic Acid Gel Stain (ThermoFisher Scientific). Equal amounts of un-reacted, cisplatin-reacted and transplatin-reacted RNA with or without Dicer digestion were loaded into each gel.

Cell based assays. Human ovarian cancer OVCAR-3 cells (ATCC HTB-161) were grown in RPMI-1640 medium supplemented with 10\% FBS (Atlanta Biologicals) and 1\% antibiotic-antimycotic solution (Mediatech-Cellgro) at $37^{\circ} \mathrm{C}$ in a humidified atmosphere with $5 \% \mathrm{CO}_{2}$ and sub-cultured when reached $\sim 80 \%$ confluence. Cells were plated at a density $\sim 10^{6}$ cells/well in 6-well plates, incubated for 24 hours and subsequently treated with cisplatin or transplatin at $5 \mu \mathrm{M}$ for 24 hours. Single concentration point $5 \mu \mathrm{M}$ and 24 hour exposure were selected, because they were reasonably expected to exert biological effects, while not being high enough to induce massive cell death and distort the results of expression analysis. For comparison, the median GI50 of cisplatin in OVCAR-3 cells is $\sim 3 \mu \mathrm{M}$ for 48 hour exposure (data from CellMiner, https://discover.nci.nih. gov/cellminer/loadDownload.do). After the treatment, cells were harvested and relative expression of mature miR-200b in cisplatin or transplatin treated vs untreated cells was determined by qPCR TaqMan miRNA assay for miR-200b and RNU6B as an endogenous reference (Applied Biosystems) as previously described [12]. For each condition, concentration of miR-200b (miR-200b signal) was expressed as $1000 \times 2^{(-\Delta C t)}$, where $\Delta C_{t}$ value represents threshold cycle for miR-200b cDNA amplification normalized to RNU6B cDNA. Each condition was replicated 3 times and results are expressed as means \pm SEM. Standard errors were calculated from replicated $C_{t}$ values by error propagation. Significance of differences between mean $\Delta \mathrm{C}_{\mathrm{t}}$ values was tested using Student's t-test. 


\section{Results}

Cisplatin interacts with pre-miR-200b and disrupts its structure differently than transplatin. The derivative melting curve of pre-miR-200b reacted with cisplatin (CP) $(\mathrm{miR} 200 \mathrm{~b}+\mathrm{CP})$ substantially differs from the curves corresponding to transplatin (TP)-reacted (miR200b+TP) and un-reacted pre-miR200b (Figure 1). The difference is most prominent in the reduction of peaks corresponding to the structural transitions at $\sim 63^{\circ} \mathrm{C}$ and $\sim 90^{\circ} \mathrm{C}$ in the pre-miR$200 \mathrm{~b}$. The result suggests that cisplatin reacts with pre-miR$200 \mathrm{~b}$ and disrupts base pairing between complementary segments of its hairpin structure. In contrast, transplatin under the same conditions did not induce structural changes in pre-miR-200b that were detectable by the melting analysis.

Dicer digestion is inhibited in pre-miR-200b reacted with cisplatin but not with transplatin. Platination of pre-miR-200b with cisplatin inhibited its subsequent digestion by Dicer and the production of a short RNA fragment, which was visible upon digestion of un-reacted (lanes 2)

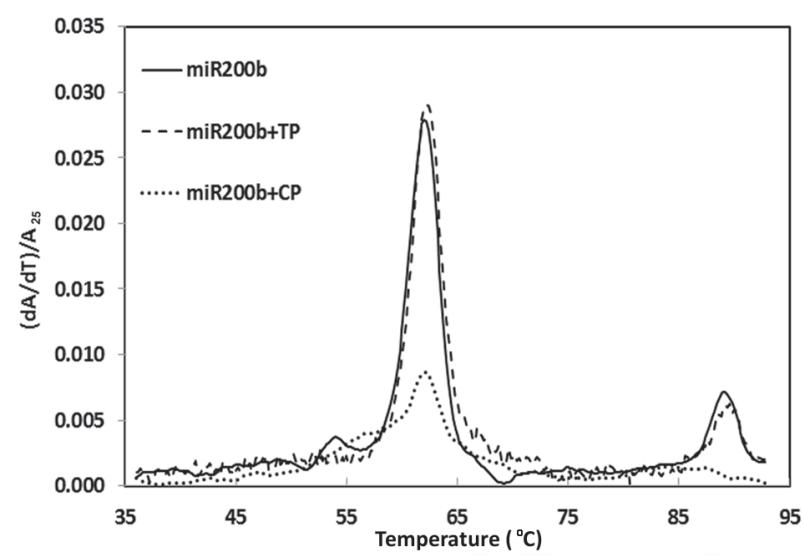

Figure 1. Derivative melting curves of the $95 \mathrm{nt}$ pre-miR-200b RNA and its cisplatin-reacted $(\mathrm{miR200b}+\mathrm{CP})$ and transplatin-reacted $(\mathrm{miR200b}+\mathrm{TP})$ products. and transplatin-reacted (lanes 6) pre-miR-200b (Figure 2A and $2 \mathrm{~B}$ ). Likewise, the two independent Dicer digestions resolved by PAGE under native (Figure 2A) and denaturing (Figure 2B) conditions, displayed strong signal for undigested pre-miRNA in cisplatin-treated pre-miR-200b (lanes 4) but nearly complete digestion of pre-miRNA in un-reacted (lanes 2) and transplatin-reacted (lanes 6) samples. This result suggests that the modification of pre-miR-200b by cisplatin but not transplatin can be functionally significant, since it inhibits the processing of platinated pre-miR200b to mature miR-200b in vitro.

Cisplatin but not transplatin decreases expression of mature miR-200b in ovarian cancer OVCAR-3 cells. Inhibition of Dicer-mediated production of mature miR-200b from cisplatin-modified pre-miR-200b motivated our effort to examine the effect of cisplatin on the expression of miR-200b in cancer cells. For this experiment we selected ovarian cancer cell line OVCAR-3, because cisplatin and its second generation analog carboplatin are instrumental drugs for the treatment of ovarian cancer, and available data show high expression of miR-200b specifically in this cell line (Supplemental Material, Figure S3). The results of our qPCR expression analysis demonstrate significant down-regulation of $\mathrm{miR}-200 \mathrm{~b}$ in cisplatin-treated vs untreated OVCAR-3 cells $(p=0.05)$, while treatment with transplatin did not show significant decrease in expression of miR-200b ( $\mathrm{p}=0.55)$ (Figure 3). This experiment was performed under conditions that did not show induction of cell death upon treatment with cisplatin or transplatin (Supplemental Material, Figure S4). Consequently, cisplatin appears to reduce cellular levels of miR-200b even at relatively low exposures, and the reduced expression of miR-200b is not likely attributable to a non-specific transcriptional shutdown associated with cell death.

\section{Discussion}

Cisplatin is an important anticancer drug whose mode of action and resistance mechanisms are complex and not entirely understood. In addition to genomic DNA, other
Figure 2. Replicated Dicer digestion reactions: products were resolved on a $12 \%$ native PAGE $(\mathrm{A})$ or $12 \%$ PAGE/8M Urea (B) and stained with Sybr Gold. (1) pre-miR-200b without Dicer, (2) pre-miR-200b with Dicer, (3) cisplatin/pre-miR-200b without Dicer, (4) cisplatin/pre-miR-200b with Dicer, (5) transplatin/pre-miR-200b without Dicer, (6) transplatin/pre-miR-200b with Dicer, (L) DNA Ladder 25 bp. The same amount of RNA was used in all wells and the differences in RNA band intensities prior to Dicer digestion (lanes $1,3,5)$ are likely due to the influence of cisplatin and transplatin bound to the pre-miR-200b on Sybr Gold binding.
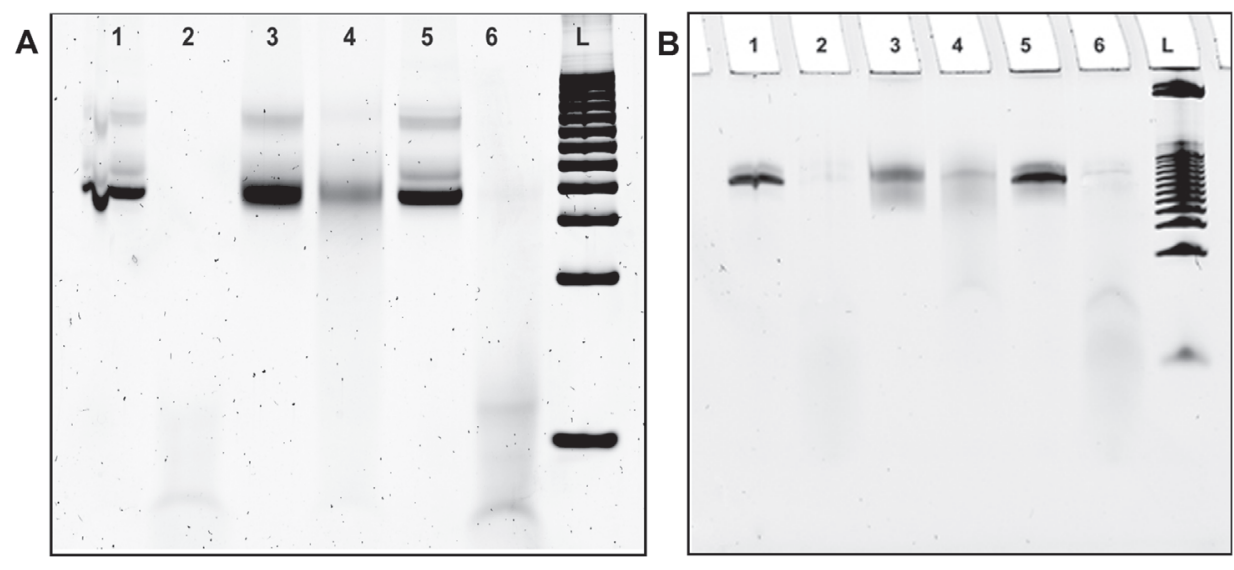
biomolecular targets have been previously implied in its anticancer activity or drug toxicity (reviewed in [2]). These possible targets included various RNA species [13], such as rRNAs [14], tRNAs [15], and snRNAs [16, 17].

We have previously suggested the possibility that cisplatin may affect a broad range of cellular processes through platination of endogenous pre-miRNAs and mature miRNAs [2]. Platination and subsequent functional impairment of these important regulators of gene expression may occur through several hypothetical mechanisms. Considering the fact that platination of RNAs blocks their degradation by various exonucleases and endonucleases [18] we hypothesized, that platination of pre-miRNAs may impair their processing by Dicer to corresponding mature miRNAs and consequently de-regulate their expression in cisplatin-treated cells. Our results support this hypothesis through altered melting profile of cisplatin-reacted pre-miR-200b, inhibition of its digestion by Dicer and decreased expression of miR-200b in cisplatin-treated ovarian cancer cells OVCAR-3.

Our finding of changed melting profile of cisplatin-reacted pre-miR-200b is consistent with the previously reported ability of cisplatin to destabilize mature miRNA mimics [19] and provides evidence for a direct interaction between cisplatin and a biologically important pre-miRNA. The difference, which we observed between cisplatin and transplatin in their reaction with pre-miR-200b is interesting, given that these structurally similar compounds substantially differ in their anticancer activities [20]. This result provides additional insight into understanding the chemical-biological differences between cisplatin and transplatin, which were known to include different deprotonation of their monoaquated species [21], formation of different DNA adducts [22, 23], and different reactivities of these compounds with various non-DNA biomolecules [2].

Inhibition of processing of cisplatin-reacted but not transplatin-reacted pre-miR-200b by Dicer provides additional evidence for cisplatin-induced structural modification of pre-miR-200b detected by our RNA melting analysis. In addition, it demonstrates that modification of pre-miR200 b by cisplatin is biologically significant and further supports the existence of biological/pharmacological differences between cisplatin and transplatin. Consistent with these results, we observed reduced expression of miR-200b in cisplatin-treated but not in transplatin-treated ovarian cancer OVCAR-3 cells. Although demonstrated in a single cell line, this result is significant in the context of the results of RNA melting and Dicer digestion experiments. It displays cisplatin-mediated deregulation of miR-200b and suggests mechanistic role of direct modification of pre-miR-200b by cisplatin in this deregulation.

MiR-200b is an important member of the miR-200 family, with demonstrated tumor-suppressive function mediated via inhibition of epithelial-to-mesenchymal transition (EMT), repression of stem cell renewal, and regulation of cell cycle progression (reviewed in [5]). Moreover, decreased expres-

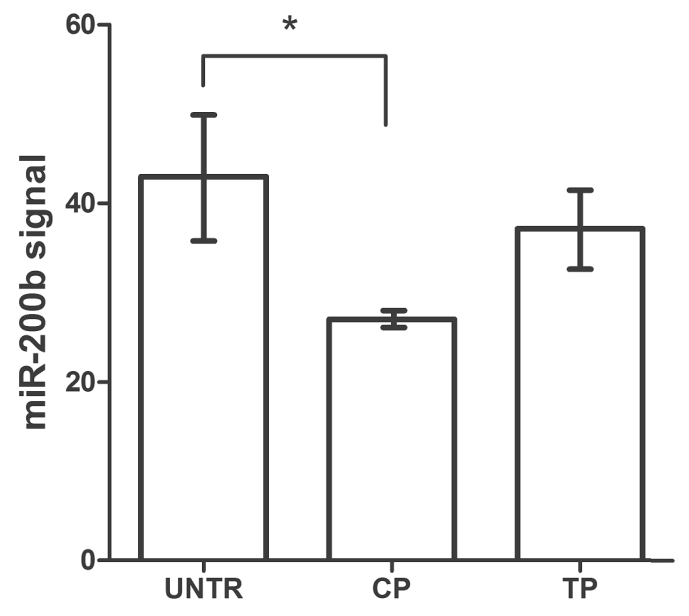

Figure 3. Expression of mature miR-200b in OVCAR-3 cells treated with cisplatin (CP), transplatin (TP) or untreated control (UNTR). Error bars: SEM; ${ }^{\star}: \mathrm{p}=\mathbf{0 . 0 5}$

sion of miR-200b was associated with drug resistance in various cancer cell types (reviewed in [24]). A specific example of this association is represented by cisplatinresistant esophageal adenocarcinoma cell line, which shows reduced expression of miR-200b relatively to isogenic cisplatin-sensitive OE19 cells (Supplemental Material, Figure S5). Therefore, it is reasonable to propose that the anticancer activity of cisplatin may be self-limited due to its interaction with pre-miR-200b followed by decreased levels of tumorsuppressive miR-200b in cisplatin-treated cancer cells. This reasoning is supported by epithelial-to-mesenchymal transition, increased migratory/invasive potential and acquisition of stem cell-like phenotype observed in various cancer cells upon treatment with cisplatin [25-27]. Thus, restoration of miR-200b or repression of its relevant target genes combined with cisplatin may be a valuable intervention in some cell contexts where treatment with cisplatin alone shows this self-limited anticancer activity. The critical importance of cell context is only visible in the light of the complexity of the role of miR-200b in cancer. This is because some reports surprisingly demonstrated oncogenic role of miR-200b in certain circumstances [28-30]. Similarly, other specific miRNAs have also been shown to display oncogenic or tumor-suppressive effects depending on cell context [31]. Consistent with the importance of cell context, the association between miR-200b expression and cisplatin sensitivity appears to be different for different cancer types (Supplemental Material, Figure S6).

To conclude, we selected pre-miR-200b because of the well-established role of miR-200b in the suppression of EMT. Since pre-miR-200b contains multiple binding sites for cisplatin (Supplemental Material, Figure S1C) [18], we hypothesized that this precursor of miR-200b can interact with cisplatin, which may result in decreased expression of miR-200b and explain previously observed EMT, stem cell- 
like phenotype and enhanced invasiveness of some cancer cells treated with cisplatin [25-27]. While EMT after cisplatin treatment has been demonstrated by other investigators, our results provide an explanation of how cisplatin treatment can lead to EMT through platination and structural/ functional impairment of pre-miR-200b. We report for the first time that cisplatin (but not transplatin) directly interacts with a pre-miRNA, impairs its processing by Dicer to mature miRNA, and down-regulates its expression in cancer cells. Treatment with cisplatin has been previously shown to change expression of numerous miRNAs [32], but evidence of their direct interactions and functional alterations by cisplatin has not yet been reported. Our finding suggests that pre-miR-200b and other pre-miRNAs with oncogenic or tumor-suppressor functions may be relevant targets of cisplatin. These interactions may be responsible for cisplatin's anticancer activity or act as modulators of cell response to this important drug.

Supplementary information is available in the online version of the paper.

\section{References}

[1] FUERTES MA, CASTILLA J, ALONSO C, PEREZ JM. Cisplatin biochemical mechanism of action: from cytotoxicity to induction of cell death through interconnections between apoptotic and necrotic pathways. Curr Med Chem 2003; 10: 257-266.

[2] MEZENCEV R. Interactions of cisplatin with non-DNA targets and their influence on anticancer activity and drug toxicity: the complex world of the platinum complex. Curr Cancer Drug Targets 2015; 14: 794-816.

[3] JANSSON MD, LUND AH. MicroRNA and cancer. Mol Oncol 2012; 6: 590-610. doi: 10.1016/j.molonc.2012.09.006

[4] GRIFFITHS-JONES S, SAINI HK, VAN DONGEN S, ENRIGHT AJ. miRBase: tools for microRNA genomics. Nucleic Acids Res 2008; 36: D154-158. doi: 10.1093/nar/gkm952

[5] FENG X, WANG Z, FILLMORE R, XI Y. MiR-200, a new star miRNA in human cancer. Cancer Lett 2014; 344: 166-173. doi: 10.1016/j.canlet.2013.11.004

[6] RHODES LV, MARTIN EC, SEGAR HC, MILLER DF, BUECHLEIN A et al. Dual regulation by microRNA-200b$3 p$ and microRNA-200b-5p in the inhibition of epithelialto-mesenchymal transition in triple-negative breast cancer. Oncotarget 2015; 6: 16638-16652. doi: 10.18632/oncotarget. 3184

[7] GIBBONS DL, LIN W, CREIGHTON CJ, RIZVI ZH, GREGORY PA et al. Contextual extracellular cues promote tumor cell EMT and metastasis by regulating miR-200 family expression. Genes Dev 2009; 23: 2140-2151. doi: 10.1101/ gad.1820209

[8] HUMMEL R, HUSSEY DJ, HAIER J. MicroRNAs: predictors and modifiers of chemo- and radiotherapy in different tumour types. Eur J Cancer 2010; 46: 298-311. doi: 10.1016/j. ejca.2009.10.027
[9] FENG B, WANG R, SONG HZ, CHEN LB. MicroRNA-200b reverses chemoresistance of docetaxel-resistant human lung adenocarcinoma cells by targeting E2F3. Cancer 2012; 118: 3365-3376. doi: 10.1002/cncr.26560

[10] BOWMAN JC, AZIZI B, LENZ TK, ROY P, WILLIAMS LD. Preparation of long templates for RNA in vitro transcription by recursive PCR. Methods Mol Biol 2012; 941: 19-41. doi: 10.1007/978-1-62703-113-4_3

[11] GROEBE DR, UHLENBECK OC. Characterization of RNA hairpin loop stability. Nucleic Acids Res 1988; 16: 1172511735 .

[12] MEZENCEV R, MATYUNINA LV, JABBARI N, MCDONALD JF. Snail-induced epithelial-to-mesenchymal transition of MCF-7 breast cancer cells: systems analysis of molecular changes and their effect on radiation and drug sensitivity. BMC Cancer 2016; 16: 236. doi: 10.1186/s12885-016-2274-5

[13] CHAPMAN EG, HOSTETTER AA, OSBORN MF, MILLER AL, DEROSE VJ. Binding of kinetically inert metal ions to RNA: the case of platinum(II). Met Ions Life Sci 2011; 9: 347-377.

[14] HOSTETTER AA, OSBORN MF, DEROSE VJ. RNA-Pt adducts following cisplatin treatment of Saccharomyces cerevisiae. ACS Chem Biol 2012; 7: 218-225. doi: 10.1021/ cb200279p

[15] PAPSAI P, SNYGG AS, ALDAG J, ELMROTH SK. Platination of full length tRNA(Ala) and truncated versions of the acceptor stem and anticodon loop. Dalton Trans 2008: 52255234. doi: 10.1039/b719542g

[16] HOSTETTER AA, CHAPMAN EG, DEROSE VJ. Rapid cross-linking of an RNA internal loop by the anticancer drug cisplatin. J Am Chem Soc 2009; 131: 9250-9257. doi: $10.1021 /$ ja809637e

[17] SCHMITTGEN TD, JU JF, DANENBERG KD, DANENBERG PV. Inhibition of pre-mRNA splicing by cisplatin and platinum analogs. Int J Oncol 2003; 23: 785-789.

[18] CHAPMAN EG, DEROSE VJ. Enzymatic processing of platinated RNAs. J Am Chem Soc 2010; 132: 1946-1952. doi: 10.1021/ja908419j

[19] ALSHIEKH A, CLAUSEN M, ELMROTH SK. Kinetics of cisplatin binding to short $\mathrm{r}(\mathrm{GG})$ containing miRNA mimics - influence of $\mathrm{Na}(+)$ versus $\mathrm{K}(+)$, temperature and hydrophobicity on reactivity. Dalton Trans 2015; 44: 12623-12632. doi: 10.1039/c5dt00663e

[20] ARIS SM, FARRELL NP. Towards Antitumor Active transPlatinum Compounds. Eur J Inorg Chem 2009; 2009: 1293. doi: 10.1002/ejic.200801118

[21] MILLER SE, HOUSE DA. The hydrolysis products of cisdichlorodiammineplatinum(II) 3. Hydrolysis kinetics at physiological pH. Inorg Chim Acta 1990; 173: 53-60. doi: 10.1016/S0020-1693(00)91054-5

[22] LIPPERT B. Trans-diammineplatinum(II): what makes it different from cis-DDP? Coordination chemistry of a neglected relative of cisplatin and its interaction with nucleic acids. Met Ions Biol Syst 1996; 33: 105-141.

[23] KASPARKOVA J, MARINI V, BURSOVA V, BRABEC V. Biophysical studies on the stability of DNA intrastrand cross-links of transplatin. Biophys J 2008; 95: 4361-4371. doi: 10.1529/biophysj.108.138909 
[24] FENG B, WANG R, CHEN LB. Review of miR-200b and cancer chemosensitivity. Biomed Pharmacother 2012; 66: 397-402. doi: 10.1016/j.biopha.2012.06.002

[25] LATIFI A, ABUBAKER K, CASTRECHINI N, WARD AC, LIONGUE C et al. Cisplatin treatment of primary and metastatic epithelial ovarian carcinomas generates residual cells with mesenchymal stem cell-like profile. J Cell Biochem 2011; 112: 2850-2864. doi: 10.1002/jcb.23199

[26] LIU YQ, ZHANG GA, ZHANG BC, WANG Y, LIU Z et al. Short low concentration cisplatin treatment leads to an epithelial mesenchymal transition-like response in DU145 prostate cancer cells. Asian Pac J Cancer Prev 2015; 16: 1025-1028.

[27] BARIBEAU S, CHAUDHRY P, PARENT S, ASSELIN E. Resveratrol inhibits cisplatin-induced epithelial-to-mesenchymal transition in ovarian cancer cell lines. PLoS One 2014; 9: e86987. doi: 10.1371/journal.pone.0086987

[28] YONEYAMA K, ISHIBASHI O, KAWASE R, KUROSE K, TAKESHITA T. miR-200a, miR-200b and miR-429 are oncomiRs that target the PTEN gene in endometrioid endometrial carcinoma. Anticancer Res 2015; 35: 1401-1410.
[29] ZHANG Z, LANZ RB, XIAO L, WANG L, HARTIG SM et al. The tumor suppressive miR-200b subfamily is an ERG target gene in human prostate tumors. Oncotarget 2016; 7 : 37993-38003. doi: 10.18632/oncotarget.9366

[30] MENG F, HENSON R, LANG M, WEHBE H, MAHESHWARI $S$ et al. Involvement of human micro-RNA in growth and response to chemotherapy in human cholangiocarcinoma cell lines. Gastroenterology 2006; 130: 2113-2129. doi: 10.1053/j.gastro.2006.02.057

[31] AGUDA BD, KIM Y, PIPER-HUNTER MG, FRIEDMAN A, MARSH CB. MicroRNA regulation of a cancer network: consequences of the feedback loops involving miR-17-92, E2F, and Myc. Proc Natl Acad Sci U S A 2008; 105: 1967819683. doi: 10.1073/pnas.0811166106

[32] BIERSACK B. Interactions between anticancer active platinum complexes and non-coding RNAs/microRNAs. Non-Coding RNA Res 2016: 1-17. doi: 10.1016/j. ncrna.2016.10.001 